ArtefaCToS. Revista de estudios de la ciencia y la tecnología

eISSN: 1989-3612

Vol. 7, No. 1 (2018), 2a Época, 75-97

DOI: http://dx.doi.org/10.14201/art2018717597

\title{
Internet en su vertiente científica: Predicción y prescripción ante la complejidad ${ }^{*}$
}

\section{Internet in Its Scientific Branch: Prediction and Prescription in the Face of Complexity}

\author{
Wenceslao J. GONZÁLEZ \\ Universidad de A Coruña, España \\ wenceslaojgonzalez@gmail.com
}

Recibido: 10/01/2018. Revisado: 20/01/2018. Aceptado: 24/01/2018

\begin{abstract}
Resumen
De las tres principales vertientes de Internet - la científica, la tecnológica y la social-, se ha prestado escasa atención a la científica. El artículo realiza un análisis filosófico-metodológico de esa vertiente de la Red de redes. Lo hace desde el ángulo de las Ciencias de lo Artificial, que habitualmente no es considerado en la Filosofía de la Ciencia. Tiene en cuenta, además, la relevancia de las Ciencias de Internet $-\mathrm{y}$ de las disciplinas científicas que usan la Red para ampliar su campo de acción o crear novedades en sentido estricto- como Ciencias Aplicadas. Por eso, se atiende expresamente a la predicción y la prescripción, que son claves para afrontar la complejidad estructural de Internet -epistemológica y ontológica- y la complejidad dinámica, que está surcada por la historicidad, en lugar de ser una mera evolución.
\end{abstract}

Palabras clave: Internet; Ciencias de lo Artificial; futuro; pautas; complejidad; estructural; dinámica.

\footnotetext{
* Esta contribución se enmarca dentro de las líneas de investigación del Proyecto FFI201679728-P del Ministerio de Economía, Industria y Competitividad (AEI). 


\begin{abstract}
Among the three main branches of the Internet - scientific, technological, and social- the scientific area has received little attention. This paper makes a philosophico-methodological analysis of this branch of the network of networks. The approach is made from the angle of the sciences of the artificial, which is not usually considered in philosophy of science. Furthermore, it takes into account the relevance of the sciences of the Internet - and the disciplines that that use this network to enlarge their field of action or to create novelties in a strict sense- as applied sciences. Thus, the paper pays an explicit attention to prediction and prescription, which are keys in dealing with the structural complexity of the Internet - epistemological and ontological-and the dynamic complexity, which is furrowed by historicity, rather than being a mere evolution.
\end{abstract}

Keywords: Internet; Sciences of the Artificial; Future; Patterns; Complexity; Dynamic.

\title{
1. Marco temático
}

Entre las realidades contemporáneas que, hasta la fecha, han recibido escasa atención filosófico-metodológica figura Internet, cuya importancia como Red de redes es innegable. Sucede, además, que supone un reto la consideración filosófica de Internet, pues entran en liza tres grandes aspectos: la Red como actividad científica, quehacer tecnológico y realidad social. Ante este triple reto, se consideran aquí esos tres focos de atención y los tipos de análisis correspondientes. A este respecto, se da prioridad al análisis de la vertiente científica, en primer lugar, por su relevancia filosófico-metodológica; $y$, en segundo término, por estar muy poco tratada en las revistas especializadas, en comparación con las otras dos vertientes.

Temáticamente, el análisis filosófico-metodológico centra la atención en las Ciencias de lo Artificial ${ }^{1}$, puesto que son clave para entender las Ciencias de Internet. Ambos grupos de disciplinas trabajan habitualmente como Ciencias Aplicadas. De ahí que el enfoque seguido en el artículo atienda a la predicción y la prescripción, en cuanto que son dos aspectos centrales de las Ciencias Aplicadas. Además, inciden de lleno en el estudio de la complejidad estructural y la comple-

\footnotetext{
${ }^{1}$ Las Ciencias de lo Artificial se entienden aquí en el sentido de Herbert Simon, que abrió un nuevo e importante campo de investigación filosófico-metodológico, como se aprecia en Simon (1996).

No obstante, su concepción acerca de lo artificial —en el marco de las Ciencias de Diseñotiene diversas limitaciones epistemológicas, metodológicas y ontológicas. Atañen a aspectos como la racionalidad evaluativa, el papel de la historicidad y la complejidad dinámica. A este respecto, una alternativa a su modo de caracterizar la Inteligencia Artificial se ofrece en cfr. González (2017a).
} 
jidad dinámica de la Red, pues hay que anticipar el futuro posible y prescribir las pautas de actuación para resolver los problemas concretos. Porque hay problemas de Internet, como el diseńo de la nomenclatura y la arquitectura interna de la $\mathrm{Red}^{2}$, que son de fondo. Lo son ya actualmente y lo serán para el futuro.

\section{Tres focos de atención acerca de Internet}

Hay al menos tres focos relevantes para afrontar el estudio de Internet, en general, y el problema de la complejidad de la Red, en particular. En primer lugar, está el ámbito científico, que lo componen el conjunto de Ciencias que sirven de base para el desarrollo de la Red de redes como plataforma tecnológica. Esto supone mirar hacia disciplinas como la Ciencia de las Redes, la Ciencia de la Web, la Ciencia de Internet en sentido estricto, y la Ciencia de Datos (Tiropanis, Hall, Crowcroft, Contractor, y Tassiulas, 2015). En segundo término, está la esfera tecnológica, donde ha habido un crecimiento llamativo de la infraestructura de la Red de redes, que permite la realización de múltiples funciones. Su complejidad y actual estado de manejabilidad para su uso operativo plantea problemas para su ampliación futura (Yuan y Gong, 2011). En tercera instancia, está el entorno que rodea ambas facetas - la científica y la tecnológica-, que se agrupa en torno a la componente social, entendida en sentido amplio (cultural, educativa, económica, política, etc.).

Desde el punto de vista filosófico, cabe analizar los tres focos relacionados con la Red y las formas de complejidad asociadas con ellos: en el ámbito científico, en la esfera tecnológica y en la componente social de Internet. Caben así un conjunto de análisis filosóficos, diversificados en tres planos epistemológicos y metodológicos distintos —el científico, el tecnológico y el social-, pero que son complementarios en el caso de Internet. Su estudio requiere tener en cuenta los niveles ontológicos (micro, meso y macro) y los marcos temporales (plazo inmediato, corto, medio, largo y muy largo $)^{3}$.

\footnotetext{
2 "The Internet's naming and addressing architecture has widely accepted limitations. Its dated and host-centric Domain Name System (DNS) fails to keep up with the current and emerging usage models, and the overloading of the IP addresses hinders both mobility and security. As we started sifting through the large body of architectural schemes and proposals, two concerns quickly became evident. First, the independent nature of the many contributions to the field has led to the overloading of architectural terms, and to the emergence of a large body of network architecture proposals with no clear understanding of their cross similarities, and their unique properties. Second, there seems to be a growing consensus in the community on the need for designing a 'smarter' network. While such evolution is bringing new potentials and service models, the community generally lacks consistent frameworks for thinking about such models and the design implications", Khoury y Abdallah (2013, p. vii).

${ }^{3}$ Sobre los marcos temporales y su relación con la predicción científica, véase González (2015a, pp. 66, 192, 219 у 251).
} 
1) La Filosofía acerca de las Ciencias de Internet, elaborada desde una perspectiva "interna", puede analizar varios aspectos: (i) los elementos constitutivos en cuanto "Ciencias" (lenguaje, estructura, conocimiento, métodos, actividad, fines y valores); (ii) su dimensión como Ciencias Aplicadas, encaminadas por tanto a la resolución de problemas concretos; y (iii) la vertiente como Ciencias de lo Artificial, en general, y Ciencias de Diseño, en particular, puesto que no son propiamente Ciencias Sociales. Las Ciencias de Internet se caracterizan por trabajar con diseños orientados a objetivos específicos, a los que siguen una serie de procesos, para llegar a unos resultados.

2) Mediante la Filosofía sobre Internet como plataforma tecnológica, cuando es planteada desde una perspectiva "interna", interesan los factores clave de la transformación creativa de lo real, para dar lugar a algo tangible: a) el conocimiento, con sus diversas facetas - científico, específico tecnológico y evaluativo-; b) el quehacer en virtud del cual se realiza esa transformación creativa, para originar una realidad nueva (como ha sucedido con las Tecnologías de la Información y la Comunicación, que en unos casos ha ido hacia la ampliación de lo ya existente y, en otros, se ha encaminado a la generación de algo distinto); y c) el producto o artefacto, elaborado a partir del conocimiento disponible y el desarrollo del quehacer en el tiempo, siendo asumibles o razonables los costes (González, 2013a).

3) Ciertamente, la Filosofía puede reflexionar sobre la dimensión social de Internet en cuanto actividad científica y como quehacer tecnológico, puesto que los resultados científicos y los productos tecnológicos tienen consecuencias sociales. Por una parte, este tipo de análisis lo hace la Filosofía de la Ciencia en su perspectiva "externa"; y, por otra, lo lleva a cabo la Filosofía de la Tecnología en su perspectiva "externa". Buscan, respectivamente, los nexos de la actividad científica y del quehacer científico con el resto de la experiencia humana. Habitualmente estos estudios están englobados dentro de la denominación de Ciencia, Tecnología y Sociedad (González, 2005).

Hasta la fecha, Internet ha sido objeto de estudio desde diversos ángulos, pero han dominado los focos de atención segundo —el tecnológico- - y tercero —el social-, con frecuencia entrelazados. Así, en los llamados "Estudios de Internet" (Internet Studies) los temas centrales son las cuestiones tecnológicas asociadas a la Red, que incluye su diseño y su desarrollo; la utilización de esa Tecnología por los creadores de contenidos y los usuarios de la Red; y los aspectos legales y políticos que inciden en el diseño de Internet o en el uso de la Red, lo que atañe a las instituciones relacionadas con Internet y la gobernanza de la Red de redes (Dutton, 2013).

Cabe, sin embargo, plantear el análisis de Internet desde un punto de vista filosófico y poner el énfasis en la perspectiva interna. Esto supone, en primer lugar, considerar diversos aspectos epistemológicos y metodológicos de la Red, además de diferentes niveles ontológicos (como micro, meso o macro), con sus 
consiguientes marcos temporales — como corto, medio o largo plazo-, que tienen especial importancia para las Ciencias Aplicadas y para la aplicación de la Ciencia ${ }^{4}$. En segundo término, comporta hacer el análisis a partir de las Ciencias de lo Artificial. Por un lado, conllevan ángulos filosófico-metodológicos distintos a los que ofrecen las Ciencias Sociales 5 ; y, por otro, son claves para poder afrontar la complejidad, tanto estructural como dinámica, en un diseńo artificial como es Internet ${ }^{6}$.

\section{Análisis filosófico-metodológico de Internet: Actividad científica, quehacer tecnológico y dimensión social}

Abordar filosóficamente la Red de redes requiere pensar en el ser y en el deber ser de Internet. A este respecto, parece claro que el desarrollo de la Red de redes genera problemas tanto de complejidad estructural como de complejidad dinámica. Esta doble complejidad incide sobre todo como Ciencia Aplicada, esto es, para poder anticipar adecuadamente el futuro posible y las pautas de actuación adecuadas para resolver los problemas concretos planteados (a corto, medio o largo plazo). A este respecto, el análisis se puede centrar en los aspectos epistemológicos y metodológicos, además de los ontológicos, sabiendo que todos ellos están en un dominio de lo artificial con proyección social.

$\mathrm{Al}$ reflexionar filosóficamente acerca del ser de Internet, hay que partir de una configuración estructural de Internet, donde interviene la creatividad científica y la innovación tecnológica ${ }^{7}$. Es una configuración que no es estática o sincrónica, ni es una mera síntesis de elementos científicos y tecnológicos, pues tiene una dinámica sometida a cambios en términos de historicidad ${ }^{8}$, con una relación bidireccional —a su vez — con el entorno social. Porque la configuración "interna" de la Red de redes no está ciertamente aislada, puesto que está entrelazada con un poderoso entramado de factores "externos", como son los usuarios (individuos o grupos, organizaciones, etc.), los condicionantes económicos de las empresas, las disposiciones normativas (nacionales o internacionales), etc.

\footnotetext{
${ }^{4}$ Sobre la distinción entre Ciencia Aplicada y aplicación de la Ciencia, cfr. Niiniluoto (1993, 1-21; en especial, pp. 9-10 y 19); y González (2013a, 11-40; en especial, pp. 12, 17-18, 25 y 27-28).

${ }^{5}$ Para análisis desde las Ciencias Sociales, relacionados con el futuro de Internet, véase Winter y Ono (2015a).

${ }^{6}$ Inciden en las Ciencias de Internet aspectos relacionados con la racionalidad y la predicción, como sucede, por ejemplo, en Ciencias de lo Artificial como la Economía. Sobre este caso, véase González (2008b).

${ }^{7}$ En esa relación entre creatividad científica y la innovación tecnológica, que es de interacción dinámica, tiene un papel relevante la Inteligencia Artificial, cfr. González (2017a, 401-403).

8 Sobre la distinción entre "procesos", "evolución” e "historicidad”, cfr. González (2013b, 299311; en especial, pp. 304-307). Acerca de la caracterización de la historicidad, González (2011).
} 
Así pues, el primer foco de atención —el científico- está en interacción dinámica con el segundo — el tecnológico—y el tercero —el social—, de modo que la configuración "interna" de Internet como actividad científica artificial (principalmente epistemológica y ontológica) sustenta innovaciones tecnológicas de la Red. A su vez, ambas influyen en repercusión social de Internet, que es enorme ${ }^{9}$, que incide en su dinámica a través del tiempo, modulando sus cambios. Porque ciertamente influye en su situación actual y el provenir, toda vez que la dinámica de la Red de redes supone un entrelazamiento entre su configuración - la plataforma tecnológica con apoyo científico- y los variables factores de entorno (sociales, culturales, económicos, políticos, etc.) ${ }^{10}$.

Ahora bien, es una relación bidireccional, porque no solo incide en la dirección que va de lo interno a lo externo — de la creatividad científica a la repercusión social, pasando por la innovación tecnológica一, sino que también va en la otra dirección. Porque los usarios de la Red, las empresas — privadas o públicas- que tienen relación con Internet, los Estados que establecen regulaciones, los agentes transnacionales que tienen un papel en la gobernanza de la Red de redes, etc., están en una interacción dinámica con la plataforma tecnológica y, por ende, con el soporte científico que la hace $\operatorname{crecer}^{11}$. Esta triple relación —actividad científica, quehacer tecnológico y dimensión social— es clave para el análisis de la complejidad estructural de Internet y para el estudio de la complejidad dinámica de la Red de redes.

Dentro de ese marco tripartito y bidireccional, si el énfasis del análisis filosófico-metodológico se sitúa en la configuración misma de Internet, entonces cabe tomar dos direcciones principales: (i) analizar las Ciencias que sirven de base para el desarrollo de la plataforma tecnológica de Internet (como la Ciencia de las Redes $^{12}$, la Ciencia de la Web, la Ciencia de Internet en sentido estricto, y la Ciencia de Datos [Tiropanis, Hall, Crowcroft, Contractor, y Tassiulas, 2015]), o (ii) estudiar la Red de redes como Tecnología, que está sometida a constante innovación (principalmente como Tecnología de la Información y la Comunicación). La primera dirección incide de modo más directo en elementos epistemológicos y

\footnotetext{
${ }^{9}$ La relevancia social que tiene Internet es clara: "The Internet is inextricably intertwined with almost every sector of society, increasing its complexity and bringing forth numerous opportunities and challenges. It has been only 50 years from its earliest conception in the early 1960 s, to its present state as a vast, interconnected network of networks spanning much of the globe and linking approximately 2.7 billion people, representing $39 \%$ of the world's population, by the end of 2013", Winter y Ono (2015b, 1).

${ }^{10}$ Uno de los aspectos involucrados es la dinámica empresarial, que — desde un punto de vista general- se aborda en Ata y Perks (2014).

${ }^{11}$ El conocimiento tecnológico requiere de modo habitual el conocimiento científico, además de otros tipos de conocimiento, como son el específico tecnológico y el evaluativo, que atañe a los fines seleccionados y a los medios a elegir. Cfr. González (2013a, 19-20 y 22).

${ }^{12} \mathrm{La}$ Ciencia de las Redes afronta problemas relacionados con la complejidad estructural relacionada con la información y la comunicación vía Internet. Véase, por ejemplo, Halldórsson (2014).
} 
metodológicos, mientras que la segunda tiene una mayor permeabilidad para el protagonismo de los usuarios de Internet (ciudadanos, organizaciones, Estados, etc.) y las empresas que elaboran contenidos para la Red (Google, Facebook, Yahoo, etc.).

Esas direcciones científica y tecnológica para enfocar la Red son complementarias en la práctica, toda vez que hay una clara interacción dinámica entre ellas ${ }^{13}$, puesto que la racionalidad científica y la racionalidad tecnológica se complementan. Lo hacen, además, con la mediación de la racionalidad económica ${ }^{14}$, que tiene también un papel importante en Internet (en los agentes que la usan, en las empresas que la promueven, en los gobiernos — como la Unión Europea- que la regulan, etc. $)^{15}$. Así, los avances científicos impulsan innovaciones tecnológicas en Internet y, paralelamente, la plataforma tecnológica de Internet requiere el apoyo de diversas Ciencias para desarrollar la Red de redes.

Sucede que, hasta la fecha, la reflexión sobre Internet realizada a tenor de la innovación tecnológica — el segundo foco de atención-, suele ser considerada habitualmente —salvo los tecnólogos vinculados a las Tecnologías de la Información y la Comunicación o a estudios afines - a partir de su consiguiente faceta ciudadana y empresarial, que es el tercer foco de atención. Así, el impacto social de la Red ha recibido mucha más atención que el enfoque de la vertiente científica. Por eso, aquí el análisis filosófico-metodológico se centrará más en la ruta que atiende al futuro de Internet a partir de las Ciencias Aplicadas, vistas en el contexto de la complejidad.

\section{Vertiente científica desde las Ciencias de lo Artificial}

Para abordar filosóficamente la vertiente científica de la Red de redes, hay que prestar especial atención a las Ciencias de lo Artificial. (i) En ellas se ubica el amplio campo temático abierto por Internet, en cuanto terreno de las Ciencias de Diseńo, de modo que, entre otros, incluye los desarrollos de la Inteligencia Artificial que sirven de punto de apoyo para nuevos objetivos, procesos y resultados en la Red (González, 2007b). (ii) Hay un conjunto de disciplinas científicas (como la Economía, la Documentación o la Comunicación) que tienen, de hecho, una relación estrecha con Internet en su vertiente científica. Porque los continuos avances que tienen estas disciplinas son, sobre todo, en su dimensión artificial

\footnotetext{
${ }^{13}$ Guarda relación esta interacción científico-tecnológica con el papel de los valores, donde Ciencia y Tecnología son como "dos piernas de un cuerpo cuando camina", por utilizar la metáfora de Nicholas Rescher (1999).

${ }^{14}$ La mediación entre la racionalidad científica y la racionalidad tecnológica es a través de la racionalidad económica. Cfr. González (1998a).

${ }^{15}$ En el caso de la Tecnología, como se pone de relieve en Internet, tienen un peso especial los valores económicos. Sobre este tema, cfr. González (1999).
} 
(esto es, de ampliación de las posibilidades humanas) ${ }^{16}$. Estos avances en décadas recientes han sido, en gran medida, gracias al apoyo de Internet (como los incesantes desarrollos de Economía Financiera, las novedades en la recuperación de la información en la Red o las nuevas formas de comunicación audiovisual de acceso inmediato y gratuito).

En primer lugar, en su vertiente científica, Internet se basa en diseños — con frecuencia, de Inteligencia Artificial-, que permiten nuevos objetivos, procesos y resultados. Con ellos se llega habitualmente a novedades, que — según sea el grado de novedad logrado — han sido catalogadas como "evolucionarias" o como "revolucionarias". Pero que, en rigor, son la expresión de la historicidad de lo artificial, en su búsqueda constante de ampliar las posibilidades humanas y de dar soluciones a problemas concretos planteados. En segundo término, Internet es el soporte para el desarrollo de disciplinas científicas basadas en diseños que, como los casos de la Economía, la Documentación o la Comunicación, potencian las posibilidades humanas y son claves en la Sociedad del Conocimiento, dentro de esta etapa histórica que Luciano Floridi (2016) llama "Hiperhistoria" (Hyperhistory).

Considerado en términos filosófico-metodológicos, el avance en las Ciencias de lo Artificial —conceptual, práctico, social e institucional - es distinto del progreso de las Ciencias Sociales. Porque son diferentes sus objetos de estudio y sus problemas y, por ende, sus métodos, que dan lugar a resultados propios, con consecuencias de índole distinta (unas guardan relación directa con aspectos opcionales humanos, mientras que las otras están más relacionadas con necesidades humanas). Así, aun cuando las Ciencias de lo Artificial puedan analizar, buscan sobre todo sintetizar, para potenciar las posibilidades humanas en el campo de lo artificial ${ }^{17}$. Pero las Ciencias de lo Artificial no forman un bloque homogéneo, en la medida en que hay dos tipos diferentes de investigación científica en este campo.

Existe una primera concepción de Ciencias de lo Artificial, que es la opción más relevante para Internet: las Ciencias de Diseño. Hay, además, una segunda acepción, que es el estudio científico-natural o científico-social de los productos o artefactos. Este es el caso de las Ciencias de los Materiales, que están inicialmente orientadas a investigar las propiedades físicas o químicas de los productos o artefactos. Pero que también pueden indagar las consecuencias sociales del uso de esos productos o artefactos, esto es, los efectos para la vida social debidos a la obsolescencia o el deterioro de los materiales, que pueden generar polución o insalubridad.

\footnotetext{
${ }^{16}$ Un rasgo característico de las Ciencias de lo Artificial, en cuanto Ciencias de Diseńo, es la potenciación de las posibilidades humanas, al ser hechura humana (buman made) orientada hacia nuevos objetivos. Cfr. Simon (1996, 3-4).

${ }^{17}$ Que las Ciencias de lo Artificial son, ante todo, Ciencias sintetizadoras más que analizadoras está realzado en Simon (1996, 4-5).
} 
Intervienen de lleno la predicción y la prescripción en la concepción de las Ciencias de Diseńo, que es la considerada primordialmente por Herbert Simon. La razón es clara: las Ciencias de Diseño se configuran habitualmente como Ciencias Aplicadas y enlazan con la aplicación de la Ciencia en contextos variables (entornos sociales, culturales, económicos, etc.). Este es el caso, por un lado, de las "Ciencias de Internet" (como la Ciencia de las Redes, la Ciencia de la Web, la Ciencia de Internet en sentido estricto, y la Ciencia de Datos); y, por otro, de las Ciencias como la Economía, la Documentación o la Comunicación, que utilizan Internet para su desarrollo, con novedades importantes respecto de etapas anteriores ${ }^{18}$.

Estos dos grupos de Ciencias tienen que afrontar problemas concretos, en cuanto que son Ciencias Aplicadas (Niiniluoto, 1993), para lo cual elaboran diseños encaminados hacia ciertos objetivos. Estos objetivos sirven de base para unos procesos, con el deliberado afán de lograr unos resultados. Esto es lo que sucede con las Ciencias configuradoras de Internet - el primer grupo-y con las Ciencias que usan la Red de redes (el segundo grupo). Así, para la configuración interna de Internet, pesan sobre todo las Ciencias de Diseño, mientras que las Ciencias Sociales tienen su cometido para aspectos "externos" de la Red, que son los relacionados con su impacto social, cultural y de poder. Pero esos factores inicialmente externos pueden ser "interiorizados" en la Red, de modo que pueden dar lugar a nuevos objetivos (el crecimiento de las Ciencias de la Web se puede explicar desde esta perspectiva).

Los resultados obtenidos sirven para resolver, reformular o descartar el problema inicial planteado. Esto sucede en Economía como Ciencia de lo Artificial (p. ej., en desarrollos de Economía Financiera y Contabilidad, en especial en la vertiente electrónica), en Documentación (p. ej., en la recuperación de la información sobre libros, archivos electrónicos, documentos en papel, etc.) y en Comunicación, tanto en formato texto como audiovisual. El cambio, sobre todo en Comunicación, ha sido revolucionario, propiciado por los desarrollos en Internet, que han potenciado el carácter artificial de la comunicación y el intercambio de información (Graham, 1999). Se aprecia en los servidores de correo electrónico, YouTube, Facebook, Snapchat, Twitter, etc.

\footnotetext{
${ }^{18}$ Hay una Economía basada en Internet (Internet Economy), para realizar transacciones en un tiempo muy breve o a entidades localizadas a gran distancia. Algunos autores, como Jaron Lanier, especialmente conocido por su trabajo acerca de la realidad virtual, considera que son los seres humanos y no los algoritmos lo que debe estar en el centro de la Economía de Internet (Lanier, 2017).
} 


\section{El reto de la predicción y la prescripción}

Característica central de las Ciencias de lo Artificial, en cuanto Ciencias Aplicadas, es que combinan la predicción y la prescripción. Porque, para la solución de problemas concretos - como son, de modo habitual, los relacionados con Internet o los correspondientes a las disciplinas que utilizan la Red para su desarrollo-, hace falta primero predecir. En efecto, sobre la base del conocimiento actual, hay que anticipar el futuro posible, para después poder abordarlo. La predicción hace falta para el diseño científico, que se orienta hacia unos objetivos, antes de desarrollar unos procesos, en la búsqueda de unos resultados ${ }^{19}$. En el caso de la vertiente científica de Internet admite tres grandes posibilidades:

a) Cuando se trata de Internet como Red de redes, cabe estimar la viabilidad, dentro de un marco temporal definido, de la ampliación o potenciación de las posibilidades humanas. b) Al tratar de disciplinas que usan Internet para ampliar su campo o para propiciar nuevos territorios temáticos, se puede enunciar lo esperable en ellas. Esto es lo que sucede en los terrenos económicos, documentales o comunicativos. c) A partir de la emergencia de propiedades en la propia Red, es posible asimismo anticipar el futuro posible, como acontece con los nuevos fenómenos generados por Internet en cuanto tal (que estudia, por ejemplo, la Ciencia de Datos) ${ }^{20}$.

Después, tras realizar la predicción —a plazo inmediato, corto, medio, largo o muy largo plazo - viene la prescripción (González, 1998b). Porque hace falta establecer unas pautas (patterns) encaminadas a la resolución de problemas concretos, dentro de un número finito de pasos. Porque la Ciencia Aplicada mira su acierto en la resolución de problemas prácticos (González, 2015a). Pueden ser cuestiones de la Red, problemas relacionados con las disciplinas científicas que la usan (económicos, documentales o comunicativos) o asuntos originados por las propiedades emergentes de la propia dinámica de Internet. A este respecto, cada Ciencia relacionada con la Red necesita anticipar y, luego, guiar la acción para la solución de problemas concretos.

Puede tener grados muy diversos de fiabilidad la anticipación del futuro, como sucede con los conceptos de "previsión", "predicción” y "pronóstico", que expresan unos grados de control muy diferentes acerca de las variables relacionadas con el futuro ${ }^{21}$. Por tanto, indican niveles de fiabilidad muy diferentes

\footnotetext{
${ }^{19}$ Dentro de las Ciencias de lo Artificial, la predicción presenta, en principio, tres facetas filosófico-metodológicas sucesivas. (i) Puede ser un objetivo destacado de las teorías vinculadas a diseños, que necesitan las predicciones antes de hacer prescripciones. (ii) Comporta procesos - procedimientos y métodos - para hacer avanzar el conocimiento científico. Ahí pueden intervenir factores teóricos, empíricos y heurísticos. (iii) Sirve como criterio de evaluación de los enunciados científicos propuestos. Su contenido — teórico, empírico y heurístico- ha de ser utilizado en los criterios prácticos de resolución de problemas concretos en liza. Cfr. González (2007a).

${ }^{20}$ Sobre la Ciencia de Datos, véase Cao (2017a) y Cao (2017b).

${ }^{21}$ Una exposición de estos conceptos y un análisis de su relación con ocho influyentes filósofos de
} 
acerca del conocimiento del provenir a corto, medio o largo plazo. A su vez, la prescripción guarda relación con el planeamiento o la planificación, que es un concepto diferente de predicción. Porque planeamiento o planificación supone, de suyo, la dirección de la acción, en razón de cálculo y distribución de tiempos. La prescripción es más amplia que el planeamiento o la planificación, de modo que puede abarcar el planeamiento o la planificación, pero tiene un carácter más general.

Parte de este carácter más amplio se debe a la interdependencia entre predicción y prescripción, que puede incluir al menos tres facetas filosófico-metodológicas: la epistemológica, la metodológica y la axiológica ${ }^{22}$. Así, la prescripción es inicialmente un concepto de tipo epistemológico, en la medida en que es el contenido intelectual de las pautas prácticas para resolver problemas. Metodológicamente, la prescripción requiere considerar aplicación práctica de los procesos por los cuales cabe guiar de manera adecuada — con racionalidad práctica— los medios para lograr el fin buscado. Aquí la prescripción converge con el planeamiento o la planificación, si bien al planificar la toma de decisiones tiene un componente volitivo más claro que las pautas prescriptivas. Axiológicamente, la prescripción siempre se hace de acuerdo con algunos valores ${ }^{23}$, aquellos aspectos dignos de mérito que han de guiar la acción como fondo de la actuación práctica.

Aparece entonces el foco de la dimensión social — la perspectiva externajunto con el foco científico - la perspectiva interna—, que se hace visible al hilo de la interdependencia entre predicción y prescripción, especialmente cuando hay que afrontar problemas de complejidad, como es el caso de la Red (a la que se da forma - en un sentido u otro- mediante prescripciones). Porque la complejidad estructural de Internet y su complejidad dinámica no son en modo alguno ajenas a los factores sociales (culturales, políticos, económicos, etc.) que rodean el uso de la Red de redes.

Por un lado, la predicción se usa en las Ciencias Aplicadas — como las Ciencias de Internet - como una guía para pensar en las pautas de actuación (poli$c y$-making). Pero estas pautas tienen normalmente una dimensión social en la Red de redes, de modo que la actividad propia de Internet como tal está entrelazada con otras actividades que utilizan la Red como plataforma tecnológica de actuación. Por otro lado, la predicción es un instrumento para la puesta en práctica o implementación de las pautas de actuación (policy-making), dentro del ámbito directo de las Ciencias de Internet (la Ciencia de la Web, la Ciencia

\footnotetext{
la Ciencia se encuentra en González (2010).

${ }^{22}$ Este enfoque está desarrollado para la Economía en González (2015a, 317-341). Se puede utilizar para las Ciencias de la Comunicación, en cuanto que son Ciencias de lo Artificial que usan Internet. Esto se aprecia en los fenómenos de televisión digital, cfr. González (2008a). También permite el estudio de la televisión vía Internet conocida como Over The Top, en cuanto expresión de Ciencias Aplicadas de Diseńo que se vehicula a través de la Red.

${ }^{23}$ Sobre esto ha insistido Amartya Sen (1986).
} 
de las Redes, la Ciencia específica de Internet, ...), pero también en los casos de las disciplinas científicas que usan la Red (como Economía, Documentación o Comunicación) y de los estudios que se ocupan de las propiedades emergentes de la Red (la Ciencia de Datos).

\section{Cometido de la predicción: De la perspectiva interna de la Ciencia a la dimensión social}

Resaltan la importancia de la predicción cada uno de los tres focos de atención mencionados: el científico, el tecnológico y el social. Coinciden en que la predicción es clave para resolver los problemas de complejidad de Internet ${ }^{24}$. (i) En el caso de la vertiente científica, la predicción anticipa el futuro posible sobre la base del conocimiento disponible, que es el paso previo a la prescripción, que ofrece las pautas de actuación para resolver problemas concretos. (ii) La predicción sirve de base para el conocimiento científico que utiliza la Tecnología de Internet para su desarrollo. Así, la faceta tecnológica de la Red se nutre de predicciones científicas, que acompaña al conocimiento específico tecnológico acerca de los instrumentos y artefactos, además del conocimiento evaluativo acerca de los fines buscados. (iii) La predicción en la vertiente social de Internet depende de los desarrollos "internos" de la Red — científicos y tecnológicos—, sobre cuya base cabe anticipar los usos posibles por los agentes —individuales y sociales-, las empresas, los Estados, etc. A su vez, la propia dinámica social permite predecir demandas futuras — informativas, comunicativas, económicas, etc.- por parte de los miembros de las entidades sociales.

A tenor de la línea científica, donde tiene protagonismo la Ciencia Aplicada y la aplicación de la Ciencia, la predicción es clave en cuanto que ofrece conocimiento futuro posible - según el grado de control de las variables disponibles-, de modo que sirve de guía para orientar la actuación de la prescripción, que ha de proporcionar las pautas de actuación para resolver problemas planteados. Estas cuestiones las atiende según el nivel de complejidad estructural y dinámica de la Red, que es creciente en las dos facetas.

Donde hay más nexo entre lo "interno" y lo "externo" es en la predicción relacionada con la plataforma tecnológica de la Red. Porque, debido a su mayor incidencia social que la Ciencia, la Tecnología de la Red de redes presenta un vínculo claro con el entorno. A este respecto, la predicción tecnológica ha de contribuir a hacer viable la ampliación de la Red y a dar solución a sus crecientes problemas de conectividad ${ }^{25}$. Esa predicción ha de tener en cuenta no sólo la viabilidad

${ }^{24}$ Un estudio metodológico de la predicción para abordar sistemas complejos, en general, se encuentra en Nicolis y Nicolis (2012, 149-213).

25 "With the dynamicity of link creation, deletion, and transient failures, given the current scale of the Internet, it is clearly no longer feasible to capture the precise connectivity of the Internet", Yuan y Gong $(2011,425)$. 
científica del diseño industrial, sino también la relación coste-beneficio de lo anticipado, lo que afecta a la creación de productos en Internet y a la interacción con el ámbito empresarial y de regulación pública de la Red (la gobernanza) ${ }^{26}$.

Incide aquí la dinámica de Internet, que Ch. S. Yoo mira desde los usuarios y las empresas. Así, ha de atender, en primer lugar, a los cambios en los entornos tecnológicos y económicos, tales como los incrementos en el número y diversidad de los usuarios de Internet, los cambios en la naturaleza de la utilización de Internet, la diversificación de las Tecnologías de transmisión y los mecanismos de usos vinculados a fines, y el aumento en la complejidad de las relaciones de negocios. En segundo término, ha de considerar las variaciones en términos de pautas públicas (policy), como los cambios debidos a la mayor heterogeneidad en las preferencias de consumo, la disminución de la gobernanza informal, la incorporación de nuevas funciones en el núcleo de la Red, el aumento en la complejidad para poder establecer precios, la presencia creciente de intermediación (incluyendo la legal), la maduración de las empresas, etc. (Yoo, 2012).

Eje de esta trayectoria intelectual para el análisis del futuro de Internet es interno-externo: va de la perspectiva interna de la creatividad científica a la dimensión social, pasando por la innovación tecnológica. Pero cabe pensar la propuesta alternativa de Jenifer Winter y Ryota Ono acerca del futuro de la Red, que es externa-interna. Consiste en mirar la plataforma tecnológica desde sus manifestaciones sociales, como son la información compartida por los agentes sociales, la relevancia de la cultura y las relaciones de poder (toda vez que se asume que tener información comporta tener poder). Estos tres aspectos constituyen el núcleo de las tres posiciones que contemplan: a) la predictiva, b) la cultural, y c) la "crítica" (que insiste en las relaciones de poder) ${ }^{27}$.

Winter y Ono entienden por "perspectiva predictiva" la visión de la plataforma tecnológica de Internet que considera clave el papel de la información para la Red de redes. Así, esta postura insiste en Internet como instrumento operativo para el depósito de información, en lugar de resaltar la repercusión sociocultural de la Red o las relaciones de poder que comporta, que conforman las otras dos opciones contempladas (Winter y Ono, 2015c, 217-224; en especial, pp. 218223). Supone concebir la información como bien de uso, esto es, ofrecen una visión externa de la información.

\footnotetext{
${ }^{26}$ Un aspecto muy importante es la neutralidad de la Red de redes. La Comisión Federal de Comunicaciones de Estados Unidos (Federal Communications Commission) decidió el 14 de diciembre de 2017 cambiar este estatuto acerca de Internet. Esta modificación tiene consecuencias muy apreciables para el futuro de la Red. Sobre esta cuestión, véase Kang (2017).

${ }^{27}$ Cfr. Winter y Ono (2015c, 217-224; en especial, pp. 218-223). Estos autores sintetizan las aportaciones del volumen que coordinan. El hilo conductor de ellas es, precisamente, la perspectiva externa acerca del futuro de Internet.
} 
Se centra la "perspectiva predictiva" en el futuro a corto plazo de Internet como "depósito de información" (reservoir of information), donde intervienen los ciudadanos, los Estados y las corporaciones. Los ciudadanos han pasado de utilizar Internet para buscar información (con frecuencia, sin coste, salvo la conexión a la Red) a ver su futuro cada vez más dependiente de Internet, lo que anticipa variaciones en las relaciones entre los usuarios y la $\operatorname{Red}^{28}$.

Asimilan de facto predicción y expectativa, de modo que, en lo esperado para el futuro próximo, figura el aumento de monitorización de la información por parte de los Estados y el incremento de los usos comerciales de Internet. Pero cabe esperar asimismo la expansión de la Red a esa parte de la población mundial — más de la mitad, a comienzos del año 2016 — que todavía no tiene acceso a Internet (sobre todo habitantes de países en vías de desarrollo) ${ }^{29}$.

Acerca de la segunda posición - la "perspectiva cultural" - Winter y Ono señalan a Internet como una fuente de innovación social y política. Así, con cierto optimismo, ven posible el reemplazamiento de una "cultura de la recuperación mecánica de la información y monitorización en secreto" por una "nueva cultura de la comunicación recíproca hecha con conciencia" (Winter y Ono, 2015c, 220). Mientras tanto, a través de la tercera opción — la "perspectiva crítica”-, consideran posible que haya unas relaciones de poder más equilibradas, de manera que Internet, entendida como "campo de las acciones cooperativas", pueda hacer que los retos locales y globales sean resueltos dentro de una civilización orientada hacia un futuro mejor desde el punto de vista social (Winter y Ono, 2015c, 222-223).

Con estos tres escenarios del futuro, vistos desde una mirada externa a la Red —el foco tres del presente artículo y a corto o medio plazo—, solo se cubre una parte del territorio futuro de Internet. Con el papel de la información, la relevancia de la cultura y las relaciones de poder se atiende a algunos aspectos del impacto de la Tecnología de Internet para los usuarios que tienen acceso a ella. En el fondo, Winter y Ono parecen plantearse Internet básicamente como Tecnología — sin atención real a las Ciencias de Internet-y su preocupación primordial da la impresión de situarse en la incidencia futura en términos de pautas públicas (policy) dentro del marco social.

\footnotetext{
${ }^{28} \mathrm{Su}$ análisis atiende preferentemente a las vertientes tecnológica y social: "Concepts discussed in future scenarios, such as 'safety Net,' 'increased Internet dependence,' 'a digital meaning society,' 'infectious connectivity,' 'split Internet,' 'autonomously governed communities, 'noosphere,' 'IoT,' and 'IoE,' indicate variations of these relationships between citizens and the Internet", Winter y Ono (2015c, 220).

${ }^{29}$ Según el fundador de Facebook, cerca de 4.000 millones de habitantes de nuestro planeta todavía no tienen acceso a Internet. Mark Zuckerberg, en el Mobile World Congress, Barcelona, 22.2.2016, en: http:/www.informationweek.com/mobile/zuckerberg-hits-mwc-to-talk-dronesai-vr/d/d-id/1324403, acceso el 8.8.2016.
} 
Pero, por un lado, hay toda una vertiente científica que es clave para el desarrollo futuro de Internet, que incide en el corto, medio y largo plazo de Internet; y, por otro lado, los actores del futuro de la Red de redes no se limitan a los ciudadanos, las empresas y los Estados, puesto que cada vez más hay actores transnacionales, como los sistemas multiagentes sobre los que ha insistido Luciano Floridi $(2016)^{30}$, cuyo protagonismo es creciente.

Esto hace que Internet aumente su complejidad estructural y que incremente su complejidad dinámica. Hace tiempo, en efecto, que Internet es más que un instrumento de información y comunicación, puesto que (i) genera nuevas formas de Ciencias de lo Artificial, (ii) contribuye al avance del conocimiento con novedosas expresiones de Tecnología — donde la Inteligencia Artificial tiene un protagonismo creciente- ${ }^{31}$, y (iii) propicia la creación de sociedades artificiales que generan un nuevo entorno social —el virtual — con incidencia en la vida social de los países.

\section{Tarea de la prescripción ante la complejidad}

Junto al cometido de la predicción, que puede ser concebida como objetivo, test y guía de la Ciencia (González, 2015, 3-46), está la tarea de la prescripción. Las Ciencias de Internet necesitan la prescripción en tanto que Ciencias Aplicadas, en general, y como Ciencias de Diseño, en particular. Hace años, Simon vio claro que el modelado de sistemas, cuando busca la solución de problemas concretos, requiere la prescripción (Simon, 1990), de modo que no se puede limitar a la predicción un modelo científico aplicado ${ }^{32}$ (aun cuando el éxito predictivo sea relevante como guía para afrontar problemas). Acertar con la prescripción adecuada — las pautas solucionadoras de problemas — se vuelve más difícil cuando se trata de sistemas complejos, principalmente al lidiar con la complejidad dinámica, como es el caso de la Red de redes.

Analizada desde un punto de vista filosófico-metodológico, en la Ciencia la prescripción comporta una faceta interna y una dimensión externa. El componente "interno" se aprecia especialmente en el plano metodológico, puesto que es un proceso imperativo-hipotético (González, 1998b, 333), a diferencia de la predicción, que es habitualmente hipotético-deductivo (aun cuando puede ser hipotético-inductivo o, en su caso, abductivo). Porque, para prescribir, hace falta indicar qué meta se desea lograr; y este objetivo requiere que se adopten los medios adecuados para alcanzar ese fin, de lo contrario la actuación no se podría considerar racional.

\footnotetext{
${ }^{30} \mathrm{El}$ marco general de su postura se encuentra en Floridi (2014).

${ }^{31}$ Un ejemplo ilustrativo es Google Translate. Cfr. Lewis-Kraus (2016).

32 Simon va más lejos, puesto que — a su juicio — la meta ha de ser dar forma al futuro y ese objetivo ha de prevalecer sobre meramente predecir el provenir. Cfr Simon (2002).
} 
Metodológicamente, los imperativos hipotéticos buscan guiar a las Ciencias de Internet y, por tanto, orientar las decisiones que se han de tomar - las elecciones entre las diversas opciones posibles- en las diversas instancias de la Red de redes. Las decisiones han de atender a los aspectos epistemológicos y ontológicos de la complejidad estructural de la Red de redes y también han de considerar la historicidad de la complejidad dinámica de Internet, sea por razones internas o por motivos externos (como, por ejemplo, los cambios en la regulación por parte de la Unión Europea, Estados Unidos o China).

Aparece entonces el papel de los valores, puesto que la toma de decisiones de las Ciencias de Internet, para prescribir las pautas de actuación a seguir, requiere una estimación de lo conveniente y lo desaconsejable hecha sobre la base de valores, que pueden ser internos — cognitivos, metodológicos, etc. - o externos (sociales, culturales, etc.). Esos valores para prescribir el curso de acción adecuado no se pueden limitar a la Ciencia Aplicada, puesto que han de contemplar también la aplicación de la Ciencia en los diversos contextos de uso ${ }^{33}$.

Primero están los valores internos de la prescripción, que dependen en cierto modo del conocimiento proporcionado por las predicciones. Porque la dirección de la acción — las pautas de actuación científica - hacia una novedad en la Red requiere conocer un objetivo que es alcanzable en un número finito de pasos. Pueden intervenir entonces diversos valores, como la accesibilidad de la meta, la consistencia de los medios, la adecuación respecto de los agentes, la relación entre el coste — no solo financiero, sino también de energías - y el beneficio, etc.

Están también los valores externos que, en el caso de la Red de redes, son particularmente importantes. Destacan, en especial, las valoraciones sociales y éticas, como la repercusión para los agentes individuales (como privacidad, respeto a la persona, etc.), los grupos sociales (en términos de trabajo, ausencia de discriminación social, capacidad de integración en el mundo laboral, etc.), las manifestaciones culturales — de mayorías y minorías, arraigadas o nuevas, etc.que promuevan el desarrollo personal y social, la neutralidad de la Red ante injerencias políticas, etc. Cabe resaltar los valores relacionados con la responsabilidad social de las empresas, para tomar decisiones que, desde un punto de vista ético, sean sostenibles para la vida social y el uso responsable del mundo virtual de la infosfera (Floridi, 2013; Floridi, 2014, 217-220).

Dos son entonces los planos de la prescripción relacionados con las Ciencias de Internet en cuanto disciplinas que amplían las posibilidades humanas. En primer lugar, están los valores relacionados con la faceta endógena de "Internet como actividad científica", esto es, de la actividad cientifica de desarrollo de la Red de redes. Entre ellos están la eficacia y la eficiencia, la búsqueda de la certeza en las pautas de actuación —y, por tanto, la disminución de la incertidumbre-, la adecuada correlación entre medios y fines — con la consiguiente mengua en el

${ }^{33}$ Hay en esto una semejanza con la Economía, cfr. González (2015a, 317-341). 
nivel de riesgo- - la competitividad ante alternativas viables, la relación asumible entre el coste - humano, social y económico- y el beneficio, la simplicidad en los procesos —o, al menos, una manejabilidad operativa asumible—, la primacía del todo sobre las partes — aunque se siga una pauta de pasos fragmentarios-, etc.

Y, en segundo término, están los valores de la faceta exógena de "Internet como actividad científica entre otras", esto es, de la actividad cientifica de Internet como una actividad humana entrelazada con otras. Aparecen entonces un amplio rango de posibilidades, a tenor de la componente entrelazada con la actividad de Internet, bien sea humana, cultural, educativa, económica, política, o de otra índole (esto es, la componente social entendida en sentido amplio). Se trata, además, de una interacción dinámica, que se despliega en el tiempo de manera creciente.

Valores de la prescripción en esta faceta exógena son los siguientes: (i) en cuanto a los agentes —individuales o sociales - en su dimensión psicosocial (confianza, fiabilidad, viabilidad, etc.); (ii) como fenómeno sociocultural de individuos, grupos, empresas, etc. (satisfacer las necesidades — de información, de comunicación, etc.-, lograr cubrir las expectativas, sintonizar con las pautas sociales, propiciar el bienestar social, etc.); (iii) en la valoración ética de carácter social (dignidad de la persona en su imagen pública, honradez en la gestión de los recursos, integridad en la gobernanza de la Red ante las presiones de entidades públicas o privadas, etc.); (iv) en cuanto al alcance político (reducir las desigualdades, incrementar la libertad en el dominio público, etc.); (v) respecto de su repercusión para el medio ambiente (protección del entorno natural, evitar daños ecológicos, etc.); ...

Sobre todo en esta faceta exógena de los valores relacionados con la prescripción en las Ciencias de Internet, donde se combinan Ciencia Aplicada y aplicación de la Ciencia, se pone de relieve el nexo entre el foco científico y el social (el primero y el tercero de los señalados al principio). Pero también laten aquí los valores propios de Internet en cuanto Tecnología, porque en la dimensión externa de la Red de redes confluyen la perspectiva externa de la Ciencia y la perspectiva externa de la Tecnología. Así, la toma de decisiones sobre el futuro de Internet ha de hacerse sobre la base de valores, que inciden tanto en la actividad científica como en el quehacer tecnológico de la Red, y donde los valores de la faceta exógena de la prescripción son especialmente importantes para la vida social.

Si prevalece la visión del futuro de Internet sobre la base de la preferencia del bien común o bien social compartido, entonces los valores de Internet como Tecnología habrían de ser complementarios de los valores de la Red como actividad científica. Esos valores - en especial, los relacionados con las Tecnologías de la Información y la Comunicación (TIC) - requerirían un tratamiento específico, a partir de los valores internos y externos de la configuración del quehacer tecno- 
lógico ${ }^{34}$. En este terreno - la Tecnología, en general, y las TIC, en particular-, los valores económicos tienen ciertamente un protagonismo mayor que en el caso de la actividad científica cuando se trata de la toma de decisiones.

Aunque el papel de lo normativo en el terreno tecnológico es más estricto, en principio, que el cometido de la prescripción en el campo científico ${ }^{35}$, hay un nexo entre los valores tecnológicos y los valores científicos. Porque hay unos valores cognitivos en la Tecnología que enlazan con los científicos, en cuanto que, en este terreno, se combina el conocimiento científico con el conocimiento específico tecnológico y el conocimiento evaluativo. Así, la Ciencia Aplicada como es, en este caso, las Ciencias de Internet - sirven de soporte para hacer diseños tecnológicos en la Red. Esto contribuye a la componente metodológica de la Tecnología, que es imperativo-hipotética. Esto supone el seguir las reglas, si se aceptan los objetivos propuestos, los medios elegidos y los costes estimados (González, 2013a, 22). Todo esto influye en el futuro de Internet, sobre todo una vez que la Ciencia predice las metas conseguibles a corto, medio o largo plazo.

Prescribir es entonces un cometido apoyado en predecir, que se torna más difícil en Internet al estar ante una complejidad doblemente dual: científica y tecnológica, estructural y dinámica. Así, ante la complejidad estructural, tanto de índole científica —epistemológica y ontológica— como de carácter tecnológico - la configuración de la plataforma de la Red, que permite su manejabilidad operativa y su conectividad-, la tarea de la prescripción es dar pautas (patterns) de actuación que solucionen los problemas actuales y los futuros.

Paralelamente, la aplicación de las pautas en el campo científico y en el terreno tecnológico ha de modularse, primero, a tenor de los distintos contextos de uso (micro, meso o macro) y, después, según una dinámica de historicidad marcada por las necesidades temporales (plazo inminente, corto, medio, largo o muy largo plazo). Esta complejidad dinámica — que no es mera "evolución" - requiere que las prescripciones se adecúen a cada caso concreto considerado, en razón de su variabilidad en términos de historicidad (González, 2017b).

\section{Coda}

Considerada Internet en su vertiente científica, ha mostrado la relevancia de la predicción y la prescripción, desde su constitución inicial como ARPANET hasta la impresionante situación actual ${ }^{36}$, que abre nuevas perspectivas de futuro.

\footnotetext{
${ }^{34}$ Este plano general de los valores tecnológicos se aborda en González (2015b).

${ }^{35}$ La mayor exigencia normativa de la Tecnología le lleva a Ilkka Niiniluoto a plantearse si es una normatividad de obligado cumplimiento, cfr. Niiniluoto (1990). Desde otro ángulo, también se ha planteado qué límites puede tener la Tecnología desde una perspectiva externa y, en tal caso, a quién le corresponde la toma de decisiones. Cfr. Niiniluoto (1997).

${ }^{36}$ Sobre la Historia de Internet, véase Berners-Lee (1999). Una interesante síntesis histórica pos-
} 
La Historia de la Red, en la que se insiste habitualmente en la Tecnología (en particular, en las Tecnologías de la Información y la Comunicación) y la Ciencia aparece en un segundo plano - hasta el punto de ser bastante recientes diversas denominaciones de las actividades científicas relacionadas con Internet-, solo se puede entender teniendo en cuenta la predicción y la prescripción. Porque ha sido necesario anticipar el futuro posible y seguir pautas para la resolución de problemas concretos, una trayectoria que generalmente ha dado buenos resultados.

Internamente la Red ha visto crecer su complejidad estructural —epistemológica y ontológica- e incrementar su complejidad dinámica de una manera incesante, sobre todo en las últimas dos décadas, debido en parte a avances en Inteligencia Artificial y al desarrollo de disciplinas científicas que, a comienzos de los años 90, apenas estaban en ciernes ${ }^{37}$. Ante el futuro, la historicidad de los procesos de predicción y prescripción permite apreciar que habrá cambios en el corto, medio y largo plazo, toda vez que Internet se ha convertido en el eje de una nueva etapa histórica: la Hiperhistoria.

Esos cambios versarán sobre los niveles ontológicos micro (los agentes individuales y grupos pequeños), meso (grupos más amplios y organizaciones con entidad propia) y macro (corporaciones, Estados, agentes transnacionales o multiagentes). Todos ellos inciden en la dimensión social - la perspectiva externa de la Ciencia, además de la perspectiva externa de la Tecnología-, pero ante todo incidirán en el tipo de los diseños científicos, las pautas de aplicación en los nuevos contextos de uso y en las innovaciones tecnológicas. Ahí cabe esperar novedades de tipo horizontal o longitudinal —una ampliación del campo cubierto- y novedades de carácter vertical o transversal (unas variaciones sustanciales o relevantes respecto de lo precedente).

\section{Referencias bibliográficas}

Ata, Nabil Abu el y Perks, Maurice J. (2014). Solving the Dynamic Complexity Dilemma. Predictive and Prescriptive Business Management: Answering the Need for a New Paradigm. Berlín: Springer.

Berners-Lee, Timothy (1999). Weaving the Web. Londres: Texere Publishing.

Cao, Longbing (2017a). Data Science: A Comprehensive Overview. En ACM Computing Surveys, 50(3), Art. 43, 1-42.

Cao, Longbing (2017b). Data Science: Challenges and Directions. En Communications of $A C M, 60(8), 59-68$.

terior se ofrece en Khoury y Abdallah (2013, 3-6).

${ }^{37}$ Conviene recordar que es el año 1989 cuando tiene lugar la creación de la World Wide Web por

Tim Berners-Lee en el CERN y que la web se puso para dominio público en 1993. 
Dutton, William H. (2013). Internet Studies: The Foundation of a Transformative Field. En Dutton, William H. (Ed), The Oxford Handbook of Internet Studies (1-27). Oxford: Oxford University Press.

Floridi, Luciano (2013). The Ethics of Information. Oxford: Oxford University Press.

Floridi, Luciano (2014). The Fourth Revolution - How the Infosphere is Reshaping Human Reality. Oxford: Oxford University Press.

Floridi, Luciano (2016). Hiperhistoria, el surgimiento de sistemas de multiagentes (SMAs) y el diseño de la infraética. Ponencia en las Jornadas sobre Inteligencia Artificial y Sociedad contemporánea: El cometido de la información, celebradas en la Universidad de A Coruña, Campus de Ferrol, presentada el 10 de marzo de 2016.

González, Wenceslao J. (1998a). Racionalidad científica y racionalidad tecnológica: La mediación de la racionalidad económica. Ágora, 17(2), 95-115.

González, Wenceslao J. (1998b). Prediction and Prescription in Economics: A Philosophical and Methodological Approach. Theoria, 13(32), 321-345.

González, Wenceslao J. (1999). Valores económicos en la configuración de la Tecnología. Argumentos de Razón Técnica, 2, 69-96.

González, Wenceslao J. (2005). The Philosophical Approach to Science, Technology and Society. En González, Wenceslao J. (Ed.). Science, Technology and Society: A Philosophical Perspective (pp. 3-49). A Coruña: Netbiblo.

González, Wenceslao J (2007a). Análisis de las Ciencias de Diseño desde la racionalidad limitada, la predicción y la prescripción. En González, Wenceslao J. (Ed.), Las Ciencias de Diseño: Racionalidad limitada, predicción y prescripción (pp. 3-38). A Coruña: Netbiblo.

González, Wenceslao J (2007b). Configuración de las Ciencias de Diseño como Ciencias de lo Artificial: Papel de la Inteligencia Artificial y de la racionalidad limitada. En González, Wenceslao J. (ed), Las Ciencias de Diseño: Racionalidad limitada, predicción y prescripción (pp.41-69). A Coruña: Netbiblo.

González, Wenceslao J. (2008a). La televisión interactiva y las Ciencias de lo Artificial. En Arrojo, María José, La configuración de la televisión interactiva: De las plataformas digitales a la TDT (pp. xi-xvii). A Coruña: Netbiblo.

González, Wenceslao J. (2008b). Rationality and Prediction in the Sciences of the Artificial: Economics as a Design Science. En Galavotti, Maria Carta, Scazzieri, Roberto y Suppes, Patrick. (Eds), Reasoning, Rationality, and Probability, pp. 165-186. Stanford: CSLI Publications. 
González, Wenceslao J. (2010). La predicción cientifica: Concepciones filosófico-metodológicas desde H. Reichenbach a N. Rescher. Barcelona: Montesinos.

González, Wenceslao J. (2011). Conceptual Changes and Scientific Diversity: The Role of Historicity. En González, Wenceslao J. (Ed.). Conceptual Revolutions: From Cognitive Science to Medicine (pp. 39-62). A Coruña: Netbiblo.

González, Wenceslao J. (2013a). The Roles of Scientific Creativity and Technological Innovation in the Context of Complexity of Science. En González, Wenceslao J. (Ed.), Creativity, Innovation, and Complexity in Science (pp. 11-40). A Coruña: Netbiblo.

González, Wenceslao J. (2013b). The Sciences of Design as Sciences of Complexity: The Dynamic Trait. En Andersen, Hanne, Dieks, Dennis, González, Wenceslao J., Uebel, Thomas y Wheeler, Gregory (Eds.), New Challenges to Philosophy of Science (pp. 299-311). Dordrecht: Springer.

González, Wenceslao J. (2015a). Philosophico-Methodological Analysis of Prediction and its Role in Economics. Dordrecht: Springer.

González, Wenceslao J. (2015b). On the Role of Values in the Configuration of Technology: From Axiology to Ethics. En González, Wenceslao J. (Ed.), New Perspectives on Technology, Values, and Ethics: Theoretical and Practical, Boston Studies in the Philosophy and History of Science (pp. 3-27). Dordrecht: Springer.

González, Wenceslao J. (2017a). From Intelligence to Rationality of Minds and Machines in Contemporary Society: The Sciences of Design and the Role of Information. Minds and Machines, 27(3), 397-424. DOI: 10.1007/ s11023-017-9439-0.

González, Wenceslao J. (2017b). Cambio conceptual y diversidad científica: El papel de la historicidad en la dinámica de la Ciencia. Factótum. Revista de Filosofía, 18, en prensa.

Graham, Gordon (1999). The Internet: A Philosophical Inquiry. Londres: Routledge.

Halldórsson, Magnus M. (ed) (2014). Structural Information and Communication Complexity: 21st International Colloquium, SIROCCO 2014. Dordrecht: Springer.

Lanier, Jaron (2017). Dawn of the New Everything: A Journey Through Virtual Reality. Londres: Bodley Head.

Lewis-Kraus, Gideon (2016, 14 de diciembre). The Great A.I. Awakening. The New York Times Magazine, pp. 7-18. Disponible en:

https://www.nytimes.com/2016/12/14/magazine/the-great-ai-awakening.html?_r=0 
Kang, C. (2017). F.C.C. Repeals Net Neutrality Rules. Disponible en: https:// www.nytimes.com/2017/12/14/technology/net-neutrality-repeal-vote.html (acceso 15. 12. 2017). Publicado en la edición en papel del diario: Kang, C., "F.C.C. Reverses Rules Requiring Net Neutrality", New York Times, 15.12.2017, page A1.

Khoury, Joud S. y Abdallah, Chaouki T. (2013). Internet Naming and Discovery: Architecture and Economics. Londres: Springer.

Nicolis, Gregoire y Nicolis, Catherine (2012). Foundations of Complex Systems: Emergence, Information and Prediction. Hackensack, NJ: World Scientific.

Niiniluoto, Ilkka (1990). Should Technological Imperatives Be Obeyed? International Studies in the Philosophy of Science, 4, 181-187.

Niiniluoto, Ilkka (1993). The Aim and Structure of Applied Research. Erkenntnis, 38, 1-21.

Niiniluoto, Ilkka (1997). Límites de la Tecnología. Arbor, 57(620), 391-410.

Rescher, Nicholas (1999). Razón y valores en la Era científico-tecnológica. Barcelona: Paidós.

Sen, Amartya (1986). Prediction and Economic Theory. En Mason, John, Mathias, P. y Westcott, J. H. (eds), Predictability in Science and Society (pp. 3-23). Londres: The Royal Society and The British Academy.

Simon, Herbert A. (1990). Prediction and Prescription in Systems Modeling. Operations Research, 38, 7-14. Compilado en Simon, Herbert A. Models of Bounded Rationality. Vol. 3: Empirically Grounded Economic Reason. Cambridge, MA: The MIT Press, 1997, pp. 115-128.

Simon, Herbert A. (1996). The Sciences of the Artificial, 3a ed. Cambridge, MA: The MIT Press.

Simon, Herbert A. (2002). Forecasting the future or shaping it? Industrial and Corporate Change, 1(3), 601-605.

Tiropanis, Thanassis, Hall, Wendy, Crowcroft, Jon, Contractor, Noshir y Tassiulas, Leandros (2015). Network Science, Web Science, and Internet Science. Communications of ACM, 58(8), 76-82.

Winter, Jenifer y Ono, Ryota (eds.) (2015a). The Future Internet: Alternative Visions. Dordrecht: Springer.

Winter, Jenifer y Ono, Ryota (2015b). Introduction to the Future of Internet: Alternative Visions. En Winter, Jenifer y Ono, Ryota (Eds), The Future Internet: Alternative Visions (pp. 1-16). Dordrecht: Springer. 
Winter, Jenifer y Ono, Ryota (2015c). Conclusion: Three Stages of the Future Internet. En Winter, Jenifer y Ono, Ryota (Eds), The Future Internet: Alternative Visions (pp. 217-224). Dordrecht, Springer.

Yoo, Christopher S. (2012). The Dynamic Internet: How Technology, Users, and Businesses are Changing the Network. Washington: AEI Press.

Yuan, Ruixi y Gong, Weibo (2011). On the Complexity and Manageability of Internet Infrastructure. Frontiers of Electrical and Electronic Engineering in China, 6(3), 424-428. 
